\title{
Association of TMEM18 variants with BMI and waist circumference in children and correlation of mRNA expression in the PFC with body weight in rats
}

\author{
Mathias Rask-Andersen ${ }^{1}$, Josefin A Jacobsson ${ }^{1}$, George Moschonis ${ }^{2}$, Rohit A Chavan ${ }^{1}$, \\ Md Abu Noman Sikder ${ }^{1}$, Elin Allzén ${ }^{1}$, Johan Alsiö ${ }^{1}$, George P Chrousos ${ }^{3}$, Yannis Manios ${ }^{2}$, \\ Robert Fredriksson ${ }^{1}$ and Helgi B Schiöth ${ }^{\star, 1}$
}

Genome-wide association studies have shown a strong association of single-nucleotide polymorphisms (SNPs) in the near vicinity of the TMEM18 gene. The effects of the TMEM18-associated variants are more readily observed in children. TMEM18 encodes a 3TM protein, which locates to the nuclear membrane. The functional context of TMEM18 and the effects of its associated variants are as of yet undetermined. To further explore the effects of near-TMEM18 variants, we have genotyped two TMEM18associated SNPs, rs6548238 and rs4854344, in a cohort of 2352 Greek children (Healthy Growth Study). Included in this study are data on anthropomorphic traits body weight, BMI z-score and waist circumference. Also included are dietary energy and macronutrient intake as measured via 24-h recall interviews. Major alleles of rs6548238 and rs4854344 were significantly associated with an increased risk of obesity (odds ratio $=1.489(1.161-1.910)$ and $1.494(1.165-1.917)$, respectively), and positively correlated to body weight $(P=0.017, P=0.010)$ and waist circumference $(P=0.003, P=0.003)$. An association to energy and macronutrient intake was not observed in this cohort. We also correlated food intake and body weight in a food choice model in rats to Tmem18 expression in central regions involved in feeding behavior. We observed a strong positive correlation between TMEM18 expression and body weight in the prefrontal cortex (PFC) $(r=0.5694, P=0.0003)$ indicating a potential role for TMEM18 in higher functions related to feeding involving the PFC.

European Journal of Human Genetics (2012) 20, 192-197; doi:10.1038/ejhg.2011.176; published online 28 September 2011

Keywords: obesity; TMEM18; FTO

\section{INTRODUCTION}

Genome-wide association (GWA) studies have led to the identification of several loci in the human genome containing genetic variants conferring an increased risk of developing overweight and obesity. ${ }^{1-2}$ One such gene to be associated with a higher BMI, the fat mass and obesity-associated gene (FTO) was identified by Frayling et al in 2007. ${ }^{3}$ FTO has since been shown to be involved in regulation of feeding behavior via homeostatic hypothalamic pathways, ${ }^{4-6}$ and its obesityassociated variants to confer gain-of-function by increasing gene transcription. ${ }^{7}$ The most recent meta analyses of GWA studies on obesity, which included genetic information from 249796 individuals, identified variants at a total of 32 genetic loci affecting the development of BMI. ${ }^{2}$ Single-nucleotide polymorphisms (SNPs) in the FTO gene, followed by SNPs in the proximity of TMEM18 and MC4R, have consistently given the strongest associations to obesity in large-scale GWA studies. TMEM18-associated SNPs were first found to be associated with a higher BMI in 2009 by the GIANT consortium, ${ }^{1}$ and this was confirmed in the same year by an independent group. ${ }^{8}$

Results from child cohorts have shown stronger effects of nearTMEM18 SNPs to obesity, compared with adults. Zhao et al ${ }^{9}$ found near-TMEM18 SNPs to confer the strongest effect on pediatric BMI out of 25 studied obesity-associated variants in a cohort of 6078 children of European descent. A similar effect was observed in a cohort of 2042 children and adolescents from four European countries when the effects of 17 variants were studied. The strongest effect size for BMI and sum of skin folds observed in this study was for the nearTMEM18 SNP rs6548238. ${ }^{10}$ The rs6548238 variant also had a moderate effect on waist circumference and height. However, no correction was made for multiple testing in this study. Results from this group also showed an association of rs6548238 and rs7561317 with obesity in a cohort of 1027 children from the Stockholm area, but no association to anthropomorphic traits was detected in this study. ${ }^{11}$ Results from adult cohorts have been more inconsistent. A study on 4923 adults from northern Sweden was unable to detect an association of rs6548238 to obesity, type 2 diabetes or measures of body composition and adipose distribution. ${ }^{12}$ Another study on a cohort of 6013 Chinese type 2 diabetes patients, 1087 healthy adolescents and 605 healthy adults was also unable to detect an association of the rs7561317 genotype to type 2 diabetes, waist circumference and waist-to-hip ratio. There was a trend toward an association to BMI but this did not reach statistical significance. ${ }^{13}$ In a Japanese adult cohort of 1129 obese and 1736 normal weight controls, the TMEM18-associated SNPs, rs2867125, rs6548238, rs4854344 and rs7561317, were all observed to be associated to obesity. ${ }^{14}$ The near-TMEM18 SNP rs7561317 was

${ }^{1}$ Department of Neuroscience, Functional Pharmacology, Uppsala University, BMC, Uppsala, Sweden; ${ }^{2}$ Department of Nutrition and Dietetics, Harokopio University of Athens, Athens, Greece; ${ }^{3}$ First Department of Pediatrics, Athens University Medical School, Aghia Sophia Children's Hospital, Athens, Greece

*Correspondence: Professor HB Schiöth, Department of Neuroscience, Functional Pharmacology, Uppsala University, BMC, Husargatan 3, box 593, Uppsala SE 75124, Sweden. Tel: +46 18471 4160; Fax: +46 18511 540; E-mail: Helgi.Schioth@neuro.uu.se

Received 7 April 2011; revised 16 July 2011; accepted 20 July 2011; published online 28 September 2011 
also tested for association to phenotypes of metabolic disorder in this study, owing to its previous association to type II diabetes. BMI, blood pressure, fasting plasma glucose, triglycerides, total cholesterol and HDL cholesterol were compared in obese and controls, but no association to rs7561317 genotype was observed.

TMEM18 is a highly conserved transmembrane protein containing three membrane-spanning regions containing a nuclear localization signal, as well as a coiled-coil domain, at the C-terminal. ${ }^{11}$ It most likely localizes to the nuclear membrane after translation, and was discovered to be involved in the migration of neural stem cells and neural progenitor cells toward glioma cells as part of the downstream signaling pathway of chemokine receptors. ${ }^{15}$ Expression profiling in rat and mouse showed TMEM18 to be expressed in all tissues tested. It is also abundantly expressed in the brain and in central regions including those involved in food intake. However, in contrast to, for example: FTO, NPY, POMC, AgRP or MC4R; TMEM18 has not been found to be regulated in brain regions regulating food intake. Our earlier studies found no regulation of TMEM18 to take place in the hypothalamus or brain stem during food deprivation or consumption of a palatable high-fat diet, ${ }^{11}$ this was in contrast to canonical feedingregulatory peptides such as POMC, NPY, MC4R and AgRP, ${ }^{16}$ as well as FTO. ${ }^{4,5}$ The anatomical, molecular and functional basis of how TMEM18 may be involved in regulating BMI is thus obscure.

In the present study, we genotyped two TMEM18 SNPs previously associated with BMI: rs6548238 and rs4854344, in a cohort of 2352 Greek schoolchildren containing anthropomorphic data on body weight, BMI and waist circumferences as well as dietary intake data, such as energy and macronutrient intake as determined by 24 -h recall interviews. Food preference and body weight in a rodent food choice model ${ }^{17}$ were also correlated to expression levels of Tmem 18 in several tissues involved in different aspects of feeding, such as components of the mesolimbic and mesocortical pathways: the amygdala, nucleus accumbens and hippocampus, as well as the prefrontal cortex (PFC), which is involved in behavioral inhibition and executive function.

\section{MATERIALS AND METHODS}

\section{Subjects and phenotype characterization}

The cohort of Greek children comprised 2658 schoolchildren, attending the 5th and 6th grades of primary schools (Table 1). This cohort was part of the 'Healthy Growth Study', a large-scale cross-sectional epidemiological study initiated in May 2007 as described previously. ${ }^{17,18}$ An extended letter explaining the aims of the current study and a consent form were provided to each parent who had a child in one of the primary schools participating in the study. Those parents who agreed to participate in the study gave their informed consent by signing the consent form, and provided their contact details. Body weight and height were measured in all study participants using standard procedures and equipment. Body weight was measured to the nearest $10 \mathrm{~g}$ and height was measured to the nearest $0.1 \mathrm{~cm}$ in standing position. BMI $z$-score was calculated relative to the International Obesity Task Force (IOTF) definitions. ${ }^{19}$ Subjects were categorized as normal weight and obese using IOTF BMI cut-off values equivalent to adult BMI of 24 and 30, respectively. Waist circumference was measured to the nearest $0.1 \mathrm{~cm}$ with the use of a non-elastic tape (Hoechstmass, Sulzback, Germany) around the trunk, at the level of umbilicus midway between the lower rib margin and the iliac crest, and with the subject at a standing position. Dietary intake data were obtained by trained dieticians and nutritionists by morning interviews with the children at school site for two consecutive weekdays and one weekend day, using the 24-h recall technique. All study participants were asked to describe the type and amount of foods, as well as all beverages consumed during the previous day, provided that it was a usual day according to the participant's perception. To improve the accuracy of food descriptions, standard household measures (cups, tablespoons, etc) and food models were used to define amounts when appropriate. At the end of each interview, the interviewers, who were dieticians rigorously trained to minimize interviewer effect, reviewed the collected food intake data with the respondent in order to clarify entries, servings and possible forgotten foods. The ratio of reported energy intake and predicted basal metabolic rate was used to asses underreporting of calorie intake. Basal metabolic rate was estimated according to Schofield equations, ${ }^{20}$ taking into account age, sex, and body weight and with cut-off limits developed by Goldberg et al. ${ }^{21}$

Food intake data were analyzed using the Nutritionist $\mathrm{V}$ diet analysis software (version 2.1, 1999, First Databank, San Bruno, CA, USA), which was extensively amended to include traditional Greek recipes, as described in Food Composition Tables of Greek Cooked Foods and Dishes. ${ }^{22}$ Furthermore, the database was updated with nutritional information of processed foods provided by independent research institutes, food companies and fast-food chains. DNA for genotyping was available for 2352 subjects (1311 girls and 1064 boys). Approval of the consent procedure, and to conduct the study, was granted by the Greek Ministry of National Education and the Ethical Committee of Harokopio, University of Athens.

\section{Genotyping and linkage disequilibrium analysis}

The genotyping of TMEM18 SNPs was carried out with pre-designed Taqman single-nucleotide polymorphism genotyping assays (Applied Biosystems, Foster City, CA, USA) and an ABI7900 genetic analyzer with SDS 2.2. software at the Uppsala Genome Center (http://www.genpat.uu.se/node462). The genotype call rate was $99.6 \%$. Test for deviation from Hardy-Weinberg equilibrium

Table 1 Descriptive characteristics of the cohort of 2352 Greek children, stratified according to weight status

\begin{tabular}{|c|c|c|c|}
\hline Characteristics & All & Normal weight & Obese \\
\hline Age (years) & $11.2 \pm 0.7$ & $11.2 \pm 0.7$ & $11.1 \pm 0.6$ \\
\hline Height $(\mathrm{cm})$ & $148.7 \pm 7.8$ & $147.3 \pm 7.7$ & $152.3 \pm 7.4$ \\
\hline BMI z-score & $0.9 \pm 1.2$ & $-0.01 \pm 0.8$ & $2.8 \pm 0.3$ \\
\hline BMI & $20.3 \pm 3.8$ & $20.3 \pm 3.8$ & $27.6 \pm 2.5$ \\
\hline Cholesterol (mg) & $230.9 \pm 127.7$ & $284.1 \pm 138.2$ & $226.1 \pm 120.8$ \\
\hline \multicolumn{4}{|l|}{ \% Total energy from } \\
\hline Fat & $40.9 \pm 7.3$ & $40.9 \pm 7.2$ & $40.8 \pm 7.9$ \\
\hline Carbohydrates & $45.6 \pm 8.5$ & $45.7 \pm 8.4$ & $45.2 \pm 9.2$ \\
\hline Protein & $15.6 \pm 3.5$ & $15.6 \pm 3.4$ & $16.1 \pm 3.7$ \\
\hline
\end{tabular}

Values are geometric means \pm SD. 
was performed using the Pearson's $\chi^{2}$-test $(1$ d.f), and none of the SNPs did deviate from Hardy-Weinberg equilibrium $(P>0.4)$. Haploview ${ }^{23}$ was used for linkage disequilibrium (LD) measurements according to confidence intervals (CIs)by Gabriel et al, ${ }^{24}$ as well as graphical representation of the LD structure indicated as $r^{2}$. The LD pattern was generated using HapMap data version 2, release 21 and $\mathrm{CEU}$ as analysis panel.

\section{Statistical analyses}

Association with obesity was analyzed with logistic regression and odds ratio with a 95\% CI. Associations between genotypes and phenotypes were analyzed with linear regression, assuming an additive model. Quantitative skewed variables were normalized by transformation before analysis. The models were adjusted for age, gender, BMI and length when needed. Statistical analyses were performed with PLINK (http://pngu.mgh.harvard.edu/purcell/plink/). ${ }^{25}$ Multiple testing was adjusted with the false discovery rate according to the procedure of Benjamini and Hochberg, ${ }^{26}$ and a $P$-value $<0.005$ was considered statistically significant.

\section{Power calculations}

For the case/control analyses, power calculations were carried out with the CaTS power calculator (http://www.sph.umich.edu/csg/abecasis/CaTS/ index.html). ${ }^{27}$ We had $80 \%$ power to detect association with obesity with a relative risk of 1.3 .

\section{Food choice model}

Thirty-six outbred male Wistar rats (Scanbur BK AB, Sollentuna, Sweden) were housed in standard macrolon cages at constant temperature $\left(22 \pm 1{ }^{\circ} \mathrm{C}\right)$ and humidity $(50 \pm 5 \%)$. Rats were 10 weeks old at the start of the study, and housed individually for 8 days during the food preference paradigm. Water and standard food chow (R36, Lactamin, Lidköping, Sweden) was supplied ad libitum during the entire experiment except for the 5-day food preference test, during which the animals had free access to three diets: a palatable high-fat diet and two reference diets with high amounts of either casein (high-protein diet) or maize starch (high-carbohydrate diet). Compositions of the different diets are described in Alsiö et al. ${ }^{28}$ The food was provided in bowls and weighed each day to measure the ingested amount. This model allowed the determination of the following outcomes: body weight at endpoint, total food intake during palatable diet presentation, high-fat diet preference (high-fat diet consumption divided by total intake) and consumption of standard chow. Subsequent to the 5-day food choice, the animals were kept on standard chow for 12 days; this 'washout' period was inserted to allow effects of the food choice diets on gene expression to subside. Animals were then killed by decapitation. All samples were collected immediately following decapitation. Dissection of the brain is described by Alsiö et al. ${ }^{28}$ Samples were then immersed in RNAlater (Applied Biosystems/Ambion, Austin, TX, USA) and stored at room temperature for $2 \mathrm{~h}$ before being stored at $-20^{\circ} \mathrm{C}$ until preparation. All animal procedures were approved by the Uppsala Animal Ethical Committee (ID: C 285/5) and followed the guidelines of Swedish legislation on animal experimentation (Animal Welfare Act SFS1998:56) and European Union legislation (Convention ETS123 and Directive 86/609/EEC).

\section{Generation of complementary DNA (cDNA)}

Tissues were homogenized by sonification (Branson sonifier B 15), and RNA was purified from the samples using the TRIzol method (Sigma-Aldrich, Stockholm, Sweden). ${ }^{29}$ Samples were then treated with DNase I (Roche Diagnostics, Bromma, Scandinavia) to remove residual DNA contamination. Presence of residual DNA contamination after DNase I treatment was checked by using PCR, and products were run on agarose gel. cDNA was then generated using reverse MLV reverse transcriptase (Invitrogen, Lidingö, Sweden) according to the manufacturer's specifications.

\section{Quantitative real-time PCR}

PCR reactions were run in a total volume of $20 \mu \mathrm{l}$ using Taq polymerase kits (Biotools, Madrid, Spain). Each reaction was performed in duplicate according to the manufacturer's specifications, and contained $75 \mathrm{~mm}$ Tris/HCL, $50 \mathrm{mM} \mathrm{KCl}$ and $20 \mathrm{~mm}\left(\mathrm{NH}_{4}\right)_{2} \mathrm{SO}_{4}, 4 \mathrm{~mm} \mathrm{MgCl} 2,0.25 \mathrm{~mm}$ dNTPs, 1:20 DMSO, $20 \mathrm{mU} / \mu \mathrm{l}$ Taq polymerase, $50 \mathrm{~mm}$ forward and reverse primer and 1:4 SYBR-green (Invitrogen). Reactions were run on iCycler temperature cyclers, and fluorescence was measured using MyiQ single color real-time PCR detection system. Data were analyzed using iQ5 software (BioRad, Sundbyberg, Sweden). Primer temperatures were optimized for specificity using temperature gradients and analyzing melting curves of the PCR products.

\section{Statistical analysis}

Gene expression was normalized using the geometric mean of the most stable housekeeping genes $(M<1.5)$, in accordance with the geNorm-method as previously published by Vandesompele et al. ${ }^{30}$ Statistical analysis was performed in Prism v 5.02 for Windows (GraphPad Software, San Diego, CA, USA; http://www.graphpad.com). The non-parametric test for correlation (Spearman's test, Prism v. 5.02) was used to test for correlation between mRNA expression and model parameters: body weight at the end of the food preference paradigm, total amount of food consumed, preference for the high-fat diet and the amount of chow consumed during the food preference paradigm.

\section{RESULTS}

TMEM18-associated SNPs rs4854344 and rs6548238 are associated to obesity, BMI and waist circumference but not dietary intake Cohort characteristics are displayed in Table 1. rs6548238 and rs 4854344 are located about $30 \mathrm{~kb}$ downstream of TMEM18 and in almost absolute LD with each other, $r^{2}=0.98$ (Supplementary Figure 1). Association to obesity was determined by comparing normal weight subjects $(\mathrm{BMI}<25)$ with obese $(\mathrm{BMI}>30)$. Analysis showed major alleles of rs6548238 and rs4854344 to be significantly associated with an increased risk of obesity (rs6548238: 1.489 (1.1611.910), $P=0.002$; rs4854344: 1.494 (1.165-1.917), $P=0.001$ ) (Table 2), which is in line with previous results on a cohort of 1027 children and adolescents from the Stockholm area. ${ }^{11}$ By using linear regression analysis, we also observed higher BMI (rs6548238: $P=0.006$, rs4854344: $P=0.005$ ) and waist circumference (rs6548238: $P=0.003$,

Table 2 Genotype distribution and odds ratio of two SNPs downstream TMEM18 between obese and normal-weight Greek children

\begin{tabular}{|c|c|c|c|c|c|c|c|c|c|}
\hline & & \multicolumn{5}{|c|}{ Genotype, n (\%) } & OR $(95 \% \mathrm{Cl})$ & $P$ & HWE \\
\hline rs6548238 & Normal weight & 2073 & $82(4.0)$ & $689(33.2)$ & $1302(62.8)$ & 20.6 & & & \\
\hline rs4854344 & Normal weight & 2072 & $83(4.0)$ & $686(33.1)$ & $1303(62.9)$ & 20.5 & & & \\
\hline $\mathrm{G}>\mathrm{T}$ & Obese & 271 & $9(3.3)$ & $62(22.9)$ & $200(73.8)$ & 14.7 & $1.494(1.165-1.917)$ & 0.001 & 0.591 \\
\hline
\end{tabular}

Abbreviations: $\mathrm{Cl}$, confidence interval; MAF, minor allele frequency; $\mathrm{OR}$, odds ratio.

Data are number of subjects in each group and number of subjects for each genotype (G) (\% in each group). MAF for each group is given in percentage. OR with a $95 \% \mathrm{Cl}$ was calculated assuming an additive model. Association with obesity was determined comparing subjects with normal weight $\left(\mathrm{BMI}<25 \mathrm{~kg} / \mathrm{m}^{2}\right)$ and obesity $\left(\mathrm{BMI} 30 \mathrm{~kg} / \mathrm{m}^{2}\right)$. HWE indicate $P$-values for deviation from Hardy-Weinberg Equilibrium, excluded if $P \leq 0.001$. 
Table 3 Association between two SNPs downstream TMEM18 and body weight, BMI $z$-score, waist circumference and dietary energy intake in 2352 Greek children

\begin{tabular}{|c|c|c|c|}
\hline & Beta & $95 \% \mathrm{Cl}$ & $\mathrm{P}_{\text {Add }}$ \\
\hline \multicolumn{4}{|l|}{ rs6548238 } \\
\hline Body weight (kg) & 0.063 & $0.011-0.115$ & 0.017 \\
\hline BMI $z$-score & 0.097 & $0.026-0.169$ & 0.005 \\
\hline Waist circumference $(\mathrm{cm})$ & 0.111 & $0.039-0.182$ & 0.003 \\
\hline Average dietary energy intake (kcal/day) & -0.034 & $-0.036-0.106$ & 0.343 \\
\hline Fiber (g) & -0.007 & $-0.064-0.078$ & 0.845 \\
\hline Cholesterol (mg) & 0.063 & $-0.008-0.135$ & 0.081 \\
\hline \multicolumn{4}{|l|}{$\%$ Total energy from } \\
\hline Fat & -0.239 & $-0.047-0.096$ & 0.513 \\
\hline Carbohydrates & 0.034 & $-0.106-0.036$ & 0.342 \\
\hline Protein & -0.039 & $-0.033-0.112$ & 0.289 \\
\hline \multicolumn{4}{|l|}{ rs4854344 } \\
\hline Body weight (kg) & 0.068 & $0.016-0.120$ & 0.01 \\
\hline BMI $z$-score & 0.102 & $0.031-0.173$ & 0.005 \\
\hline Waist circumference $(\mathrm{cm})$ & 0.110 & $0.039-0.182$ & 0.003 \\
\hline Average dietary energy intake (kcal/day) & -0.038 & $-0.03-0.109$ & 0.291 \\
\hline Fiber (g) & -0.003 & $-0.068-0.075$ & 0.926 \\
\hline Cholesterol (mg) & 0.065 & $-0.006-0.136$ & 0.071 \\
\hline \multicolumn{4}{|l|}{$\%$ Total energy from } \\
\hline Fat & -0.029 & $-0.042-0.101$ & 0.422 \\
\hline Carbohydrates & 0.041 & $-0.112-0.030$ & 0.261 \\
\hline Protein & -0.042 & $-0.030-0.114$ & 0.260 \\
\hline
\end{tabular}

Beta indicates transformed beta values. $P$ indicates $P$-values adjusted for significant covariates.

rs4854344: $P=0.003$ ) for carriers of the major allele of rs6548238 and rs4854344, and trends for higher body weight (rs6548238: $P=0.017$, rs4854344: $P=0.010$ ) (Table 3). The two SNPs were not associated with the average dietary energy intake (kcal/day). Similarly, daily intake of fiber and cholesterol, as well as percentages of energy intake derived from total fat and protein, were independent on TMEM18 genotypes in this cohort (Table 3). Underreporting of calorie intake was assessed for $22.6 \%$ of the entire cohort and $12.1 \%$ of the cohort for which genotype data were available. However, no effect on the outcome was observed when we removed subjects assessed to be underreporting from the analysis. This indicates that the lack of an association between food intake and TMEM18-related SNPs observed in our cohort was likely not caused by the underreporting of calorie intake by the participants.

Expression of PFC TMEM18 correlates to body weight in rats The rodent food choice paradigm was previously employed to identify associations between hypothalamic gene expression levels and food intake, as well as body weight of rats. ${ }^{28}$ We did observe expression of TMEM18 in all the tissues studied. However, we observed no correlation for TMEM18 expression with high-fat diet preference, amount of chow, amount of total food ingested or body weight in the hypothalamus, nucleus accumbens, amygdala or hippocampus. We did, however, observe a strong correlation between TMEM18 expression and body weight in the rat PFC $(r=0.5694, P=0.0003)$ (Figure 1$)$. We also investigated the expression of several proteins known to be involved in cortical signaling (Supplementary Table 1). Body weight was significantly correlated to expression of GABA-receptor subunit $\alpha-3$ (Gabra3) $(P=0.0052)$, as well as $5-\mathrm{HT}_{2 \mathrm{~A}}$ receptor $(P=0.049), \alpha-1 \mathrm{~B}$ adrenergic receptor $(P=0.0168)$ and GABA B receptor $2(P=0.0426)$ (Supplementary Figure 1).
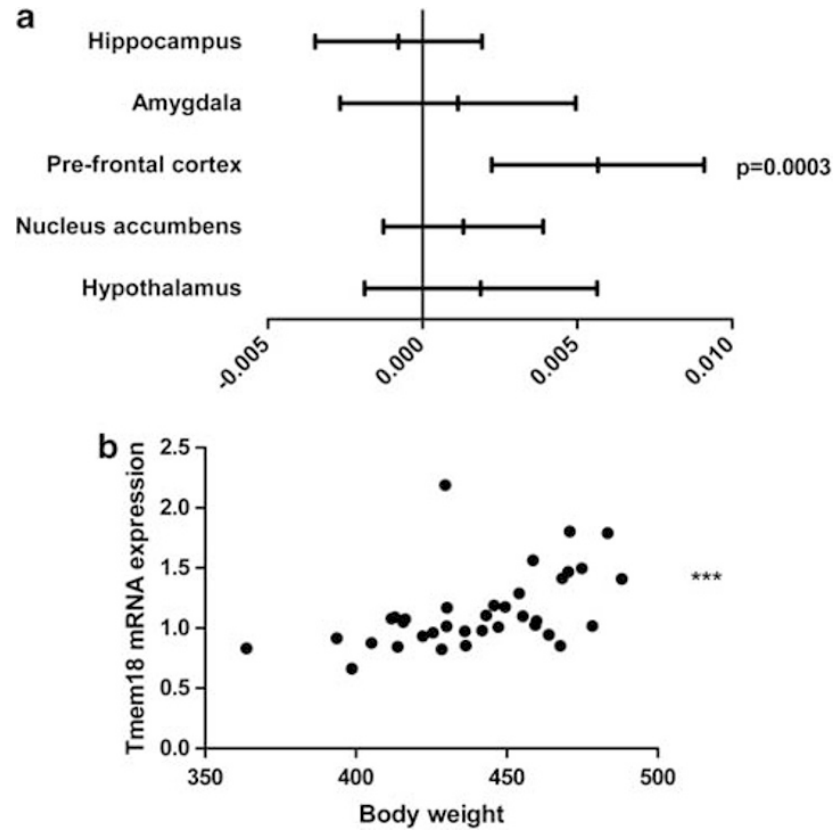

Figure 1: a Slopes for best-fit curves $\pm 95 \% \mathrm{Cl}$, generated from linear regression analysis of mRNA expression of TMEM18 in regions of the brain in rats $(n=36)$ as measured by quantitative reverse transcriptase PCR, against body weight in grams. Linear regression was performed in Prism v5.02 (GraphPad Software). b Relative expression of TMEM18 mRNA in the rat PFC, as measured by quantitative reverse transcriptase PCR, plotted against body weight in grams. Results from linear regression analysis of TMEM18 mRNA expression against body weight at the end of the food preference paradigm shows a significant correlation between body weight and expression of TMEM18. ${ }^{* *} P<0.001$.

\section{DISCUSSION}

Here, we show that the major alleles of the near-TMEM18 SNPs: rs6548238 and rs4854344, were significantly associated with an increased risk to develop obesity in the studied cohort of 2352 Greek children. These results are in line with the previous results on a cohort of children from the Stockholm area, ${ }^{11}$ as well as with the results from Zhao et al ${ }^{9}$ who observed strong associations of three TMEM18-associated SNPs to obesity in a cohort of 6078 children of European descent. We also observed associations of rs6548238 and rs4854344 to anthropomorphic traits: waist circumference, body weight and BMI $z$-score, which were not detected in the Stockholm cohort. We did however not observe any association to dietary energy and macronutrient intake (Table 3).

Studies on adult cohorts have yielded more inconsistent results indicating that the effects of TMEM18-associated variants may be more pronounced in early childhood and adolescence. This is supported by a study by Elks et $a^{31}$ who observed obesity-risk alleles identified through GWA studies, including TMEM18-associated SNPs, to confer an effect on weight gain also in early childhood and adolescence. This effect was observed as early as in the first weeks after birth. An association study for BMI-increasing alleles and birth weight reported a slight effect of rs6548238 on birth weight, but not when multiple testing was accounted for. ${ }^{32}$

rs6548238 and rs4854344 are located about $30 \mathrm{~kb}$ downstream of TMEM18. Haplotype analysis showed rs6548238 and rs4854344 to be in almost complete LD. Both SNPs are located in a haplotype block, which does not encompass the TMEM18 gene (see Supplementary Figure 2). It is thus unlikely that rs6548238 and rs4854344 are linked 
to causative SNPs inside the TMEM18 gene. They may however have an effect on the transcription of TMEM18 by affecting the binding of transcription factors or co-regulators of transcription. At this stage, the mechanisms behind the effect of these variants are, as of yet, undetermined.

We present for the first time an association of body weight in rats with gene expression of TMEM18. This association was neuroanatomically localized to the PFC. The PFC has been proposed to have an important role in integrating behavioral cues from multiple sources in the brain (reviewed by Miller and Cohen ${ }^{33}$ ). It receives information from various sources, among these are the hippocampus, hypothalamus, mesolimbic circuit, motor cortex and sensory cortices. In the context of feeding behavior, a model for the PFC in prioritization of long-term goals by inhibiting behavioral cues from the hypothalamus, brainstem and mesolimbic pathway, that is, hunger and craving, has been suggested. This has been termed 'top-down' control of, or inhibition of, behavior, which denotes higher cognitive function inhibiting behavioral cues from the basal ganglia, brain stem and hypothalamus. Other aspects of PFC function includes what has been described as impulse control and working memory. A functional role for TMEM18 in the PFC is, as of yet, unclear. Earlier reports of involvement of TMEM18s in neural migration may be a valuable lead and point to a role in the development of the PFC, or in dynamic plasticity related to memory formation and learning. Learningdependent cortical remodeling has been reported in the primate PFC in response to afferent dopamine signaling, ${ }^{34}$ and synaptic plasticity in the PFC has been shown to be modulated by monoaminergic signaling from PFC afferents. ${ }^{35}$

In this context, it is of interest that we observe concomitant correlation in the rat PFC of GABA-receptor transcripts Gabra3, Gabbr2, and monoamine receptor transcripts 5Ht2a and Adrb1, with body weight at the end of the feeding paradigm. The $\alpha_{1 \mathrm{~A}}$ adrenergic receptor and the $5-\mathrm{HT}_{2 \mathrm{~A}}$-receptor both act as excitatory $\mathrm{G}$ protein-coupled receptors coupling to the $\mathrm{G} \alpha_{\mathrm{q}}$ signaling cascade, and elicits phospholipase C-mediated $\mathrm{Ca}^{+}$release. ${ }^{36}$ Postsynaptically, they act to stimulate GABA release from cortical interneurons and also glutamate release from pyramidal neurons. ${ }^{37}$ Stimulation of $\alpha_{1}$-adrenergic receptors in the rat PFC has also been shown to increase local release of 5 -HT. ${ }^{36}$ The GABA receptor is a ligand-gated ion channel commonly composed of five subunits: two $\alpha$, two $\beta$ and one $\gamma$ subunit. Each subunit also has several subtypes, which creates a large number of combinations making up the GABA-receptor isoform diversity. The subunit composition of the receptor in turn affects ligand affinity and can also affect its electrophysiological properties. ${ }^{38}$ The $\alpha 3$ GABAreceptor subunit is frequently a component of the $\alpha_{3} \beta_{2} \gamma_{2}$ GABAreceptor subtype, which is expressed in the cerebral cortex and in monoaminergic neurons of the basal forebrain. Knockout studies have shown this subunit to be involved in sensorimotor signaling and a hyperdopaminergic phenotype, which has been suggested to be related to schizophrenia. ${ }^{39}$ The correlation of the expression of these genes in the PFC of rats with high body weight indicates PFC serotonergic and adrenergic signaling to be affected in these animals with concurrent effects on monoaminergic receptor expression and subsequent effects on GABA-receptor gene transcription.

In summary, we show that SNPs in TMEM18 are associated with obesity and body weight, as well as anthropomorphic measures of adiposity: waist circumference, in a cohort of 2352 Greek children. No association was found to behavioral phenotypes: dietary energy and macronutrient intake as measured by 24 -h recall interviews. It should be mentioned that every evaluation of dietary intake is limited, and the limitations of 24-h recalls in capturing habitual intake at the individual level are well known, because of the large day-to-day variation in intake that exists and possible difficulties in remembering what has actually been ingested. However, for the purpose of this study, three 24-h recalls on a reasonably large group, with no obvious bias, was deemed appropriate to study a plausible association with energy intake and TMEM18. However, replication of this with other estimates of energy intake would be valuable. Surprisingly, we found that the expression of TMEM18 in the rat PFC is correlated to body weight in our animal model. This is the first functional data showing a link between expression of TMEM18 and phenotype. Concomitant regulation of monoaminergic- and GABA-ergic receptor transcripts could prompt further studies for a role on TMEM18 in synaptic plasticity.

\section{CONFLICT OF INTEREST}

The authors declare no conflict of interest.

\section{ACKNOWLEDGEMENTS}

The study was supported by the Swedish Research Council, Brain Research Foundation, Novo Nordisk, Tore Nilsons foundation and Åhlens foundation. RF was supported by the Göran Gustafsson foundation. The SNP genotyping was performed by the SNP Technology Platform, Uppsala, Sweden (http:// www.genotyping.se) with support from Uppsala University and the Knut and Alice Wallenberg foundation, and at the Uppsala Genome Centre. We thank the Healthy Growth Study Group for their contribution in this study. The Healthy Growth Study Group consists of (1) Harokopio University Research Team/ Department of Nutrition and Dietetics: Yannis Manios (Coordinator), George Moschonis (Project manager), Katerina P Skenderi, Evangelia Grammatikaki, Odysseas Androutsos, Sofia Tanagra, Alexandra Koumpitski, Paraskevi-Eirini Siatitsa, Anastasia Vandorou, Aikaterini-Efstathia Kyriakou, Vasiliki Dede, Maria Kantilafti, Aliki-Eleni Farmaki, Aikaterini Siopi, Sofia Micheli, Louiza Damianidi, Panagiota Margiola, Despoina Gakni, Vasiliki Iatridi, Christina Mavrogianni, Kelaidi Michailidou, Aggeliki Giannopoulou, Efstathoula Argyri, Konstantina Maragkopoulou, Maria Spyridonos, Eirini Tsikalaki, Panagiotis Kliasios, Anthi Naoumi, Konstantinos Koutsikas, Katerina Kondaki, Epistimi Aggelou, Zoi Krommyda, Charitini Aga, Manolis Birbilis, Ioanna Kosteria, Amalia Zlatintsi, Elpida Voutsadaki, Eleni-Zouboulia Papadopoulou, Zoi Papazi, Maria Papadogiorgakaki, Fanouria Chlouveraki, Maria Lyberi, Nora Karatsikaki-Vlami, Eva Dionysopoulou and Efstratia Daskalou. (2) Aristotle University of Thessaloniki/School of Physical Education and Sports Sciences: Vassilis Mougios, Anatoli Petridou, Konstantinos Papaioannou, Georgios Tsalis, Ananis Karagkiozidis, Konstantinos Bougioukas, Afroditi Sakellaropoulou and Georgia Skouli. (3) University of Athens/ Medical School: George P Chrousos, Maria Drakopoulou and Evangelia Charmandari.

1 Willer CJ, Speliotes EK, Loos RJ et al: Six new loci associated with body mass index highlight a neuronal influence on body weight regulation. Nat Genet 2009; 41: 25-34.

2 Speliotes EK, Willer CJ, Berndt SI et al: Association analyses of 249796 individuals reveal 18 new loci associated with body mass index. Nat Genet 2010; 42: 937-948.

3 Frayling TM, Timpson NJ, Weedon MN et al: A common variant in the FTO gene is associated with body mass index and predisposes to childhood and adult obesity. Science 2007; 316: 889-894.

4 Fredriksson R, Hagglund M, Olszewski PK et al: The obesity gene, FTO, is of ancient origin, up-regulated during food deprivation and expressed in neurons of feeding-related nuclei of the brain. Endocrinology 2008; 149: 2062-2071.

5 Olszewski PK, Fredriksson R, Olszewska AM et al: Hypothalamic FTO is associated with the regulation of energy intake not feeding reward. BMC Neurosci 2009; 10: 129

6 Church C, Moir L, McMurray $\mathrm{F}$ et al: Overexpression of Fto leads to increased food intake and results in obesity. Nat Genet 2010; 42: 1086-1092.

7 Berulava T, Horsthemke B: The obesity-associated SNPs in intron 1 of the FTO gene affect primary transcript levels. Eur J Hum Genet 2010; 18: 1054-1056.

8 Thorleifsson G, Walters GB, Gudbjartsson DF et al: Genome-wide association yields new sequence variants at seven loci that associate with measures of obesity. Nat Genet 2009; 41: 18-24.

9 Zhao J, Bradfield JP, Li M et al: The role of obesity-associated loci identified in genomewide association studies in the determination of pediatric BMI. Obesity (Silver Spring) 2009; 17: 2254-2257. 
10 den Hoed M, Ekelund U, Brage $\mathrm{S}$ et al: Genetic susceptibility to obesity and related traits in childhood and adolescence: influence of loci identified by genome-wide association studies. Diabetes 2010; 59: 2980-2988.

11 Almen MS, Jacobsson JA, Shaik JH et al: The obesity gene, TMEM18, is of ancient origin, found in majority of neuronal cells in all major brain regions and associated with obesity in severely obese children. BMC Med Genet 2010; 11: 58.

12 Renstrom F, Payne F, Nordstrom A et al: Replication and extension of genome-wide association study results for obesity in 4923 adults from northern Sweden. Hum Mol Genet 2009; 18: 1489-1496.

$13 \mathrm{Ng}$ MC, Tam CH, So WY et al: Implication of genetic variants near NEGR1, SEC16B, TMEM18, ETV5/DGKG, GNPDA2, LIN7C/BDNF, MTCH2, BCDIN3D/FAIM2, SH2B1, FTO, MC4R, and KCTD15 with obesity and type 2 diabetes in 7705 Chinese. J Clin Endocrinol Metab 2010; 95: 2418-2425.

14 Hotta K, Nakamura M, Nakamura T et al: Association between obesity and polymorphisms in SEC16B, TMEM18, GNPDA2, BDNF, FAIM2 and MC4R in a Japanese population. J Hum Genet 2009; 54: 727-731.

15 Jurvansuu J, Zhao Y, Leung DS et al: Transmembrane protein 18 enhances the tropism of neural stem cells for glioma cells. Cancer Res 2008; 68: 4614-4622.

16 Lindblom J, Haitina T, Fredriksson R, Schioth HB: Differential regulation of nuclear receptors, neuropeptides and peptide hormones in the hypothalamus and pituitary of food restricted rats. Brain Res Mol Brain Res 2005; 133: 37-46.

17 Moschonis G, Tanagra S, Vandorou A et al: Social, economic and demographic correlates of overweight and obesity in primary-school children: preliminary data from the Healthy Growth Study. Public Health Nutr 2010; 13: 1693-1700.

18 Jacobsson JA, Rask-Andersen M, Riserus $U$ et al: Genetic variants near the MGAT1 gene are associated with body weight, BMI and fatty acid metabolism among adults and children. Int J Obes (Lond) 2011; e-pub ahead of print 8 February 2011; doi:10.1038/ijo.2011.11

19 Cole TJ, Bellizzi MC, Flegal KM, Dietz WH: Establishing a standard definition for child overweight and obesity worldwide: international survey. BMJ (Clinical research ed.) 2000; 320: 1240-1243.

20 Schofield WN: Predicting basal metabolic rate, new standards and review of previous work. Hum Nutr Clin Nutr 1985; 39 (Suppl 1): 5-41.

21 Goldberg GR, Black AE, Jebb SA et al: Critical evaluation of energy intake data using fundamental principles of energy physiology: 1. Derivation of cut-off limits to identify under-recording. Eur J Clin Nutr 1991; 45: 569-581.

22 Trichopoulou A: Composition Tables of Foods and Greek Dishes. 3rd Edn. Parisianou Publications: Athens, Greece, 2004.

23 Barrett JC, Fry B, Maller J, Daly MJ: Haploview: analysis and visualization of LD and haplotype maps. Bioinformatics 2005; 21: 263-265.
24 Gabriel SB, Schaffner SF, Nguyen $\mathrm{H}$ et al: The structure of haplotype blocks in the human genome. Science 2002; 296: 2225-2229.

25 Purcell S, Neale B, Todd-Brown K et al: PLINK: a tool set for whole-genome association and population-based linkage analyses. Am J Hum Genet 2007; 81: $559-575$.

26 Benjamini Y, Hochberg Y: Controlling the false discovery rate - a practical and powerful approach to multiple testing. J Roy Stat Soc B Met 1995; 57: 289-300.

27 Skol AD, Scott LJ, Abecasis GR, Boehnke M: Joint analysis is more efficient than replication-based analysis for two-stage genome-wide association studies. Nat Genet 2006; 38: 209-213.

28 Alsiö J, Roman E, Olszewski PK et al: Inverse association of high-fat diet preference and anxiety-like behavior: a putative role for urocortin 2. Genes Brain Behav 2009; 8: 193-202.

29 Rio DC, Ares M, Hannon GJ, Nilsen TW: Purification of RNA using TRIzol (TRI reagent). Cold Spring Harbor Protoc 2010, pdb prot5439.

30 Vandesompele J, De Preter K, Pattyn F et al: Accurate normalization of real-time quantitative RT-PCR data by geometric averaging of multiple internal control genes. Genome Biol 2002; 3: RESEARCH0034.

31 Elks CE, Loos RJ, Sharp SJ et al: Genetic markers of adult obesity risk are associated with greater early infancy weight gain and growth. PLoS Med 2010; 7: e1000284.

32 Kilpelainen TO, den Hoed M, Ong KK et al: Obesity-susceptibility loci have a limited influence on birth weight: a meta-analysis of up to 28219 individuals. Am J Clin Nutr 2011; 93: 851-860.

33 Miller EK, Cohen JD: An integrative theory of prefrontal cortex function. Annu Rev Neurosci 2001; 24: 167-202.

34 Kuboshima-Amemori S, Sawaguchi T: Plasticity of the primate prefrontal cortex. Neuroscientist 2007; 13: 229-240.

35 Goto Y, Yang CR, Otani S: Functional and dysfunctional synaptic plasticity in prefrontal cortex: roles in psychiatric disorders. Biol Psychiatry 2010; 67: 199-207.

36 Amargos-Bosch M, Adell A, Bortolozzi A, Artigas F: Stimulation of alpha1adrenoceptors in the rat medial prefrontal cortex increases the local in vivo 5 hydroxytryptamine release: reversal by antipsychotic drugs. J Neurochem 2003; 87: 831-842.

37 Andrade R: Serotonergic regulation of neuronal excitability in the prefrontal cortex. Neuropharmacology 2011; 61: 382-386.

38 Keramidas A, Harrison NL: The activation mechanism of alpha1beta2gamma2S and alpha3beta3gamma2S GABAA receptors. J Gen Physiol 2010; 135: 59-75.

39 Yee BK, Keist $R$, von Boehmer $L$ et al: A schizophrenia-related sensorimotor deficit links alpha 3-containing GABAA receptors to a dopamine hyperfunction. Proc Natl Acad Sci USA 2005; 102: 17154-17159.

Supplementary Information accompanies the paper on European Journal of Human Genetics website (http://www.nature.com/ejhg) 